\title{
Factores motivacionales, académicos, sociales y económicos asociados con el abandono de la educación media técnica en Medellín, Colombia*
}

\begin{abstract}
Motivational, academic, social and economic factors associated with the abandonment of technical secondary education in Medellín, Colombia
\end{abstract}

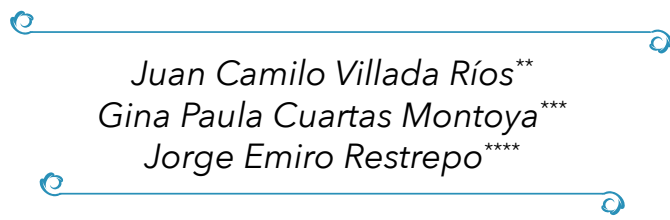

Recibido 10.09.2019• Arbitrado 25. 09. $2019 \bullet$

Aprobado 09.10. 2019

* Este artículo es producto de la tesis de maestría desarrollada por el primer autor en el marco de la Maestría en Psicología de la Educación de la Universidad Cooperativa de Colombia.

** Magister en Psicología de la Educación, Secretaria de Educación, Medellín. Contacto: jcamilovr@gmail.com ORCID: 0000-0001-9143-5787

*** Doctora en Psicología, Profesora investigadora, Facultad de Psicología, Universidad Cooperativa de Colombia-Medellín. Contacto: gina.cuartasm@campusucc. edu.co ORCID: 0000-0001-5385-1560

**** Doctor en Neuropsicología, Profesor Asistente, Facultad de Educación y Ciencias Sociales, Tecnológico de Antioquia, Medellín. Contacto: jorge. restrepo67@tdea.edu.co_ ORCID: 0000-0001-8790-7454

\section{Resumen}

El estudio tuvo como objetivo evaluar los factores motivacionales, académicos, sociales y económicos asociados con el cambio de la educación media técnica a la educación media académica en una muestra estadísticamente representativa de 83 instituciones educativas públicas de la ciudad de Medellín, Colombia. Se aplicó a 320 estudiantes un instrumento diseñado y validado para evaluar estos cuatro factores. Los dos factores más importantes asociados con el cambio de modalidad en la educación media fueron el motivacional y el académico. En el factor motivacional resaltó la falta de información y de orientación vocacional por parte de las instituciones educativas, y el desinterés por parte de los estudiantes. En el factor académico se evidenció una insatisfacción de las expectativas académicas y laborales, y la alta exigencia de la educación media técnica. El factor social y el factor económico no presentaron valores de consideración. Se discutieron los hallazgos 
a partir de la literatura relacionada con las causas locales e internacionales asociadas con la deserción escolar.

Palabras clave: educación media, abandono, desinterés escolar, expectativas académicas y laborales

\section{Abstract}

This study aimed to evaluate the motivational, academic, social and economic factors associated with the change from middle technical education to middle academic education in a statistically representative sample of 83 public educational institutions in the city of Medellín, Colombia. An instrument designed and validated to evaluate these four factors was applied to 320 students. The two most important factors associated with the change in modality in secondary education were motivational and academic. In the motivational factor highlighted the lack of information and vocational guidance by the educational institutions, and the lack of interest on the part of the students. The academic factor showed a dissatisfaction of the academic and labor expectations, and the high requirement of technical secondary education. The social factor and the economic factor did not present values of consideration. The findings were discussed from the literature related to local and international causes associated with scholar desertion.

Keywords: middle school, school dropout, school disinterest, academic and work expectations

\section{Introducción}

En el sistema educativo colombiano, la educación media se ubica entre la educación básica secundaria (de nueve grados) y la educación superior (técnica, tecnológica y profesional universitaria en pregrado y postgrado). La educación media comprende los grados décimo y undécimo y "tiene como fin la comprensión de las ideas y los valores universales y la preparación para el ingreso del educando a la educación superior y al trabajo" (Ley 115, 1994, art. 27). La educación media tiene el carácter de educación media académica y educación media técnica. El carácter académico tiene como objetivo la adquisición y profundización en conocimientos y habilidades cognitivas relacionadas con la reflexión, el pensamiento crítico, la investigación, la solución de problemas y la participación social a partir de la fundamentación y la formación en las ciencias naturales, las ciencias sociales, las humanidades y las artes. El carácter técnico tiene como propósito la preparación para el 
desempeño laboral en los sectores de la producción y los servicios a través de la formación en la teoría y la práctica desde lo más avanzado de la ciencia y la técnica (Ley 115, 1994, art. 32).

En la Ley 749 de 2002 (Art. 7) está especificado que el ingreso a los programas de formación técnica profesional en las instituciones de educación superior colombianas podrá realizarse siempre y cuando se haya cursado y aprobado la educación básica secundaria en su totalidad y se tenga más de diez y seis años o se haya obtenido el Certificado de Aptitud Profesional (CAP) expedido por el Servicio Nacional de Aprendizaje (SENA). De manera que, si el propósito del estudiante es complementar, actualizar, suplir conocimientos y formarse en aspectos académicos o laborales conducentes a la obtención de certificados de aptitud ocupacional (Decreto 4904 de 2009, cap. 1) y obtener el acceso a los programas de formación técnica profesional, no existe el requerimiento legal de que haya cursado la educación media. Así, la educación media no es un requisito para la educación para el trabajo y el desarrollo humano, que tiene como interés "promover la formación en la práctica del trabajo mediante el desarrollo de conocimientos técnicos y habilidades, así como la capacitación para el desempeño artesanal, artístico, recreacional y ocupacional..." (Decreto 4904 de 2009, cap. 1).

Como está estipulado, la educación media no es obligatoria, el Plan Nacional de Desarrollo 2014-2018 sugiere un tránsito paulatino hacia una educación media obligatoria para el 2030 (Ministerio de Educación Nacional, 2016) y no es de estricta responsabilidad del Gobierno Nacional financiar sus recursos ni establecer sus lineamientos. De hecho, se ha considerado que ha existido un olvido y una subvaloración (Sánchez, Gómez, Cadavid y Urrego, 2004) que ha generado una ruptura entre la educación básica y la educación superior. Esta ruptura, que Gómez, Díaz y Celis (2009) denominan como el "puente quebrado", ha afectado más a los estratos socioeconómicos bajos quienes reciben una educación que los está formando para el trabajo y no para que continúen con un desarrollo académico, intelectual y profesional que les permita construir un proyecto de vida sólido y permanente que no se limite a la instrumentalización ni simplemente al saber hacer (Traversa, 2013). En este punto se cuestiona la articulación de la educación media con el SENA. Según Gómez (2009. p. 16), todo este escenario está relacionado con “... la lógica pragmática de que es preferible que los estudiantes pobres tengan alguna capacitación ocupacional (...), que continuar con una educación académica general que no capacita para el trabajo y que tampoco garantiza acceso a los pocos cupos, y altamente competidos, en la educación superior pública". 
Las cifras de cobertura de este nivel del sistema educativo reflejan el complejo escenario político, económico y administrativo en el que está la educación media en Colombia. El Ministerio de Educación Nacional, en un informe del año 2003 reconoció que “... la cobertura de la media es muy baja con respecto a la meta de universalización de la educación" (p. 16). En el año 2009, las tasas de cobertura bruta y neta en la educación media fueron del $76 \%$ y 40\% respectivamente. Estos dos valores fueron los más altos desde el 2002 (Barrera-Osorio, Maldonado y Rodríguez, 2012). Entre el año 2002 y 2012, la tasa neta de cobertura aumentó del 30\% al 41\% (Ministerio de Educación Nacional, 2016a). De acuerdo el informe de la Organización para la Cooperación y el Desarrollo Económicos (OCDE) (Ministerio de Educación Nacional, 2016b), "la cobertura está aumentando permanentemente, a pesar de que los niveles de acceso y la calidad siguen siendo bastante inferiores comparados con la mayoría de países de la OCDE” (p. 215).

En Colombia, en 2014, el 33,7\% de las personas con edades entre los 19 o 20 años y el $29,3 \%$ con edades entre los 21 y los 24 años no se habían graduado de la educación media. Durante ese año, el porcentaje de personas con educación media incompleta fue de $25.7 \%$ a nivel nacional. Antioquia, ese mismo año, fue el octavo departamento en tasas de graduación de la educación media (55.7\%), por detrás de Bogotá, Atlántico, Santander, Valle del cauca, Cundinamarca, Risaralda y Quindío (García, Maldonado y Jaramillo, 2016). Además de desertar del sistema educativo en el nivel de la educación media, también se ha generado un abandono de la modalidad técnica y un tránsito hacia la media académica. Las cifras de bachilleres académicos superan a las de bachilleres técnicos. Entre 1996 y 1999, de los 3.200.000 bachilleres de todo el territorio nacional, un $68 \%$ terminó en la modalidad académica (Ministerio de Educación Nacional, 2003).

Un reporte más reciente presentado por García et al. (2016) indicó que, de un total de 6884 sedes educativas en el territorio nacional, solo un $27,9 \%$ correspondían al carácter técnico, un 13,3\% al carácter académico y técnico, y un $58,2 \%$ al carácter académico. De los 830.817 estudiantes matriculados en esas sedes educativas, solo un $37,6 \%$ estaba matriculado en la modalidad de educación media técnica. El 62.4\% restante estaba matriculado en la modalidad de educación media académica. De las 3376 sedes educativas en la región andina, solo un 22,9\% correspondían al carácter técnico, un 14,13\% al carácter académico y técnico, y un $62,7 \%$ al carácter académico. En esta misma región, de los 446.182 estudiantes matriculados en las sedes educativas, solo un 33,65\% estaba matriculado en la modalidad de educación media técnica. El 66.35\% restante estaba matriculado en la modalidad de educación media académica. 
"La deserción es un problema crónico en Colombia después de finalizar la educación primaria" (Ministerio de Educación Nacional, 2016, p. 229). Esta conclusión del Ministerio de Educación tiene cifras concretas para la educación media. El porcentaje promedio anual nacional de deserción en educación secundaria y media es de $9 \%$ para las sedes educativas con carácter técnico, de $10 \%$ para las sedes de carácter académico y de $8 \%$ que tienen los dos caracteres (García et al., 2016). Según el reporte de la Unidad de Análisis Sectorial de la Secretaría de Educación de Medellín (Alcaldía de Medellín, 2014), la tasa de deserción en la educación media fue de 3,38\% en 2012 y de 2,39\% en 2013. En este mismo informe de la Alcaldía de Medellín, se revisaron, entre 2004 y 2013, las causas más frecuentes asociadas con la deserción escolar en los niveles de básica, secundaria y media. En 2013, por ejemplo, la principal causa por la cual los estudiantes se retiraron fue por razones o motivos familiares (47,1\%). En 2004 y 2005 la principal causa había sido la situación económica (35,9\%). El desinterés por el estudio, la violencia en el sector, la lejanía del plantel educativo y el bajo rendimiento académico fueron los otros factores asociados con la deserción en ese periodo.

En la Encuesta Nacional de Deserción Escolar (Ministerio de Educación Nacional, 2011) se caracterizaron los factores asociados a la deserción en las instituciones oficiales del país. En Medellín, la encuesta se realizó con 87 establecimientos educativos y 1364 estudiantes. En cuanto a las condiciones de la zona de ubicación de los establecimientos educativos, el 37\% de los estudiantes desvinculados informó que la lejanía del plantel había sido la causa para la deserción. Respecto a las condiciones de los establecimientos educativos, el maltrato de los compañeros (18\%) y una educación distinta a los intereses educativos (15\%), fueron las principales causas. En relación con las condiciones de los hogares, el cambio de casa por el trabajo de los padres (61\%) y los problemas económicos (33\%) figuraron como las principales causas. Finalmente, en lo relativo a las condiciones individuales de los estudiantes, el informe reportó que las dificultades académicas (13\%) y el interés en una educación no formal o para el trabajo (11\%) fueron las dos primeras causas de deserción.

En general, los estudios sobre deserción escolar han sugerido factores institucionales, sociales, económicos, emocionales e intelectuales como posibles causas (Espíndola y León, 2002; Ministerio de Educación Nacional, 2011; Ruiz-Ramírez, García-Cué y Pérez-Olvera, 2014). El análisis de los factores asociados con la deserción ha sido un interés programático que ha ocupado la agenda política y administrativa de varios países de Latinoamérica en general y de Colombia en particular (Espíndola y León, 2002). Pese a que en algunos países como México (Landero, 2012), Chile (Espínola, 2011) o 
Argentina (Ferreyra et al., 2006), se ha venido analizando la problemática de la deserción en la educación media, persiste la necesitad de explorar más el problema debido a la insuficiencia de información y a las implicaciones económicas, sociales y personales que acarrea cualquier revés en esta etapa del sistema educativo.

Regularmente, los factores asociados con la deserción se han analizado en el contexto global de la educación básica y media (Ministerio de Educación Nacional, 2011) o en el contexto de la educación superior en general (Ministerio de Educación Nacional, 2009). Pero hay muy poca información relativa a los factores que causan la deserción exclusivamente en la educación media. Menos frecuentes o inexistentes, quizás, sean los estudios sobre los factores asociados con la deserción en la educación media técnica en particular. El interés de este estudio no ha sido conocer los factores asociados con la deserción escolar en el nivel de la media técnica sino conocer los factores asociados con el abandono de la media técnica y el tránsito hacia la media académica. El interés no está específicamente en el fenómeno de la deserción de la educación media sino en el fenómeno del cambio de carácter, de la técnica a la académica, esto es, el abandono de la media técnica con continuidad en la media académica.

Esta investigación, que se realizó con estudiantes de instituciones educativas públicas de la ciudad de Medellín, tuvo como objetivo describir los factores motivacionales, académicos, sociales y económicos que se asocian con el cambio de la educación media técnica a la educación media académica. Identificar, describir y analizar cada uno de estos factores favorecerá el avance hacia la comprensión de las causas que podrían explicar por qué hay un mayor interés en la media académica en particular y también, subsidiariamente, explicar por qué ocurre la deserción de la media técnica en general. Como bien lo señalan Dimas y Malagón en su análisis sobre la pertinencia de la educación media la “... pertinencia de la educación recibida en este nivel será decisiva en las oportunidades laborales, educativas y personales de los jóvenes que la transitan" (2011, p.). Es en este nivel educativo donde los estudiantes adquieren lo que Gómez, Díaz y Celis (2009) han denominado "sentido de vida". 


\section{Metodología}

\section{Diseño}

Se realizó un estudio transversal con un enfoque cuantitativo de nivel descriptivo.

\section{Participantes}

De acuerdo con el reporte del Equipo de Articulación de Media Técnica de la Subsecretaria de la Prestación del Servicio Educativo de la Secretaría de Educación de Medellín, en la ciudad, en 2016, había 120 instituciones educativas de carácter académico y técnico. De esta población se seleccionó aleatoriamente una muestra estadísticamente representativa de 83 instituciones educativas (porcentaje de error de $5 \%$, nivel de confiabilidad de $90 \%$ y distribución de las respuestas de 50\%). De estas 83 instituciones se seleccionaron 320 estudiantes que cumplieron con el criterio de inclusión. Criterio de inclusión: se seleccionaron estudiantes que estuvieran matriculados en décimo y undécimo grado de la media académica y que hubieran estado previamente matriculados en la media técnica. Es decir, se seleccionaron únicamente estudiantes escolarizados en décimo y undécimo grado que hubieran dejado la media técnica y se hubieran cambiado para la media académica.

\section{Instrumento}

Debido a la inexistencia de un instrumento de medición estandarizado que permitiera valorar los factores de interés y el tipo de población de este estudio, se decidió crear un instrumento para tal propósito. Se elaboró un cuestionario de 40 ítems con cuatro opciones de respuesta (Totalmente de acuerdo, De acuerdo, Desacuerdo, Totalmente desacuerdo). Los ítems fueron agrupados en cuatro categorías (motivacional, académica, social y económica) cada una con diez ítems (las categorías fueron comprobadas posteriormente mediante un análisis factorial confirmatorio). Las categorías fueron establecidas a partir de una revisión y análisis de literatura y los ítems derivados fueron evaluados por tres expertos en el área (un experto de la Secretaria de Educación de Medellín, un experto del SENA y un experto en educación de una universidad acreditada del país). La consistencia interna del cuestionario tuvo un Alfa de Cronbach de 0,95. El cuestionario demostró tener validez de contenido y confiabilidad (consistencia interna). 


\section{Consideraciones éticas}

La investigación se realizó cumpliendo con lo establecido en la Ley 1090 de 2006 del Colegio Colombiano de Psicólogos, que reglamenta el ejercicio de la profesión de la psicología y establece el correspondiente código deontológico y bioético.

\section{Análisis de datos}

Los datos fueron sistematizados y analizados utilizando el programa IBM SPSS v. 24. Se realizaron análisis descriptivos, además de los análisis de confiabilidad del instrumento de medición.

\section{Resultados}

El promedio de edad fue de 16,6 años para las mujeres y de 16.8 años para los hombres. La muestra estuvo conformada con porcentajes relativamente iguales en cuanto al sexo, pero fue mayor el porcentaje de mujeres $(51,6 \%$ mujeres y 48,4\% hombres). En cuanto al nivel de formación, los dos grados tuvieron porcentajes similares, pero fue mayor el grado décimo $(57,2 \%$ grado décimo y 42, 8\% grado undécimo). La jornada escolar tuvo porcentajes similares, siendo mayor el porcentaje para la jornada de la tarde (51.4\%) que el de la mañana (48,6\%). En relación con el estrato socioeconómico, el 62,9\% pertenece a estrato 2 , un $14,3 \%$ al estrado 1 , un $17,6 \%$ al estrato 3 y solo un $5 \%$ a los estratos 4 y 5 . Respecto a la composición familiar, el 58,5\% de los estudiantes convive con un solo hermano, el $27,5 \%$ con dos y un $14 \%$ convive con más de tres hermanos. Por otro lado, el 34,4\% de los participantes son el mayor de los hermanos, el $27,5 \%$ es el menor en la familia y solo un $10,2 \%$ son hijos únicos.

En relación con los ingresos económicos, en el 96,2\% de los estudiantes sus ingresos provienen de sus padres (especialmente del padre), un 3,16\% dicen que provienen de los hermanos, y un $0,63 \%$ dice que provienen de sí mismos. El 93.1\% de los estudiantes reportó no estar trabajando. Solo un 6.9\% manifestó estar trabajando en el momento de la encuesta. Sobre la participación en actividades extracurriculares, el 49,1\% contestó afirmativamente (en su mayoría, deportes) y el 51,9\% negativamente. Entre quienes respondieron afirmativamente, el 92,2\% sostiene que estas actividades no afectan su desempeño escolar mientras que el 7,8\% manifestó que sí afectaba. En la tabla I se presentan las características sociodemográficas de los participantes. 
Tabla I. Características sociodemográficas de los participantes.

\begin{tabular}{|c|c|}
\hline Característica & Porcentaje \\
\hline \multicolumn{2}{|l|}{$\overline{\text { Sexo }}$} \\
\hline Hombres & $48,4 \%$ \\
\hline Mujeres & $51,6 \%$ \\
\hline \multicolumn{2}{|l|}{ Estrato socioeconómico } \\
\hline Estrato 1 & $14,3 \%$ \\
\hline Estrato 2 & $62,9 \%$ \\
\hline Estrato 3 & $17,6 \%$ \\
\hline Estratos 4 y 5 & $5 \%$ \\
\hline \multicolumn{2}{|l|}{ Jornada escolar } \\
\hline Mañana & $48,6 \%$ \\
\hline Tarde & $51.4 \%$ \\
\hline \multicolumn{2}{|l|}{ Composición familiar } \\
\hline Padre, madre y un solo hermano & $58,5 \%$ \\
\hline Padre, madre y dos hermanos & $27,5 \%$ \\
\hline Padre, madre y tres o más hermanos & $14 \%$ \\
\hline \multicolumn{2}{|l|}{ Posición entre los hermanos } \\
\hline Mayor & $34,4 \%$ \\
\hline Intermedia & $27.9 \%$ \\
\hline Menor & $27,5 \%$ \\
\hline Hijo único & $10,2 \%$ \\
\hline \multicolumn{2}{|l|}{ Fuente de ingresos } \\
\hline Padres (especialmente el padre) & $96,2 \%$ \\
\hline Hermanos & $3,16 \%$ \\
\hline Ellos mismos & $0,63 \%$ \\
\hline \multicolumn{2}{|l|}{ Trabaja } \\
\hline Sí & $6.9 \%$ \\
\hline No & $93.1 \%$ \\
\hline \multicolumn{2}{|l|}{ Actividades extracurriculares } \\
\hline Sí & $49,1 \%$ \\
\hline No & $51,9 \%$ \\
\hline
\end{tabular}

Fuente: Propia

\section{Factor motivacional}

El 17,9\% de los estudiantes negó haber recibido en noveno grado la información relacionada con el programa de media técnica. El 29,3\% manifestó no haber recibido información sobre orientación vocacional y el programa 
técnico de su institución. Un 15,7\% de los estudiantes dijo no haber sido informado sobre los compromisos evaluativos que se adquiría en la media técnica durante el grado $10^{\circ}$ y $11^{\circ}$. El 15,6\% de ellos expresó no haber sido informado de los compromisos disciplinarios que se adquiere en la media técnica durante el grado $10^{\circ}$ y $11^{\circ}$. Un $25,6 \%$ de ellos manifestó no haber ingresado a la media técnica voluntariamente. El 12,5\% de los estudiantes afirmó haber recibido influencias de un compañero de su institución educativa para iniciar la media técnica sin que ésta fuera de su interés. El 12,2\% de los estudiantes declaró que inició la técnica por influencia familiar. Un $15,6 \%$ de los estudiantes afirmó haber iniciado la media técnica por haberse sentido obligado por las directivas de la institución educativa. El 4,3\% sostuvo haberse retirado por influencia externa, como la familia. El 25,7\% manifestó haberse retirado de la media técnica por iniciativa propia y sin influencia externa.

\section{Factor académico}

El 20,3\% de los estudiantes sostuvo que se retiró por que la media técnica no cumplió sus expectativas académicas. El 17,8\% se retiró porque no cumplió sus expectativas laborales. Un 27,2\% manifestó haberse retirado por no haber querido ser técnico en el programa que se ofrecía en la institución educativa. El 9,6\% de los estudiantes manifestó haberse retirado por faltas de asistencia en el programa técnico. El 7,8\% informó que se retiró por su conducta y disciplina. Un 10\% declaró haberse retirado porque no entendía las indicaciones de los docentes. El 15\% de los estudiantes manifestó haberse retirado por tener problemas para comprender las competencias o los contenidos en el programa técnico. El 15,3\% de los estudiantes declaró haberse retirado por su rendimiento académico. Un 17,4\% informó que se retiró porque su rendimiento académico general en el bachillerato se afectó negativamente. El 17,5\% manifestó que se retiró porque la exigencia académica fue demasiado alta.

\section{Factor social}

El 6,8\% de los estudiantes informó que se retiró de la media técnica porque tenía que madrugar mucho a la institución educativa. Un 1,5\% dijo que se retiró porque sus papás no terminaron el bachillerato. El 1,6\% manifestó que se retiró de la media técnica porque sus compañeros lo acosaban. Un 1,2\% se retiró por ser demasiado joven para estudiar. El 1,5\% de los estudiantes declaró que se retiró porque en el barrio no es bien visto que estudie tanto tiempo. Un 1,5\% dijo que se retiró por motivos de violencia. El 1,6\% afirmó que se retiró 
por estar esperando un bebé. Un 2,8\% de los estudiantes manifestó que se retiró porque en la familia le insistieron en salirse del programa. El 1,9\% dijo que se retiró de la media técnica porque los amigos del barrio le dijeron que estudiar no era importante. Un 2,2\% se retiró por no poder caminar solo en su barrio a altas horas de la noche.

\section{Factor económico}

El 2,8\% de los estudiantes afirmó que se retiró de la media técnica porque la institución educativa no le proporcionaban el transporte para estar en la contra jornada. Un 4,8\% afirma que se retiró porque la institución educativa no le proporcionaban la alimentación para estar en la contra jornada. El 1,6\% manifestó que se retiró por ser demasiado adulto para estar en un aula. Un $1 \%$ de los estudiantes dice que se retiró de la media técnica por ser demasiado joven para pensar en funciones laborales. El 2,4\% dijo haberse retirado porque necesitaba trabajar por razones personales. Un $2,2 \%$ se retiró porque en la familia le indicaron que después de la jornada escolar tenía que trabajar. El 5,9\% afirmó que se retiró por no tener los materiales solicitados. El 3,8\% de los estudiantes declaró que se retiró porque no tenía almuerzo. El 3,5\% dijo que se retiró porque no tenía los pasajes. Un 11,2\% afirmó que se retiró porque salía muy tarde de la institución educativa. En la Tabla II se resumen los principales ítems de cada factor según su porcentaje.

\section{Tabla II. Principales items de cada factor según su porcentaje}

\begin{tabular}{llc}
\hline Factor & Ítem & $\%$ \\
\hline Motivacional & No haber recibido información sobre orientación vocacional y el & 29,3 \\
& programa técnico de su institución & \\
& Iniciativa propia y sin influencia externa & 25,7 \\
& No haber ingresado voluntariamente & 25,6 \\
& No haber recibido en noveno grado la información relacionada con el & 17,9 \\
& programa de la media técnica & 27,2 \\
Académico & No haber querido ser técnico en el programa que se ofrecía en la & \\
& institución educativa & 20,2 \\
& La media técnica no cumplió sus expectativas académicas & 17,8 \\
& La media técnica no cumplió sus expectativas laborales & 17,5 \\
& La exigencia académica fue demasiado alta & 17,4 \\
& Su rendimiento académico general en el bachillerato se afectó & \\
& negativamente & 15,0 \\
& Problemas para comprender las competencias o los contenidos en el & \\
\hline & programa técnico. &
\end{tabular}




\begin{tabular}{llc}
\hline Social & Tenía que madrugar mucho a la institución educativa & 6,8 \\
& En la familia le insistieron en salirse del programa & 2,8 \\
& No poder caminar solo en su barrio a altas horas de la noche & 2,2 \\
Económico & Salía muy tarde de la institución educativa & 11,2 \\
& No tener los materiales solicitados & 5,9 \\
& La institución educativa no le proporcionaban la alimentación para & 4,8 \\
& estar en la contra jornada \\
\hline
\end{tabular}

Fuente: Propia

\section{Análisis y discusión}

En el factor motivacional, el ítem más relevante fue el relacionado con la orientación vocacional y la información sobre el programa técnico de la institución. El 29,3\% de los estudiantes que se retiró de la media técnica manifestó no haber recibido orientación ni información al respecto. El segundo ítem más importante de este factor fue el hecho de que un 25,7\% de los estudiantes se retiró por iniciativa propia y sin influencia externa. Finalmente, un $25,6 \%$ de ellos manifestó no haber ingresado a la media técnica voluntariamente. Estos porcentajes son relevantes porque están implicando a uno de cada tres (para el primer ítem) y dos de cada cuatro (en los otros dos ítems) de los estudiantes que se retiraron de la media técnica.

Según Espíndola y León (2002), la falta de interés por los estudios es uno de los factores asociados con la deserción escolar. En Chile, Nicaragua, Paraguay y Perú entre un $10 \%$ y un $25 \%$ de los adolescentes de ambos sexos manifestaron la falta de interés como razón principal del retiro del sistema educativo. En la República Dominicana y Venezuela los porcentajes estuvieron entre un $25 \%$ y un $48 \%$. Esta falta de interés podría explicar por qué hasta un $25,7 \%$ de los estudiantes se retiró por iniciativa propia y sin influencia externa, teniendo en cuenta que los factores sociales y económicos, que obtuvieron porcentajes muy bajos, no podrían explicarlo. Aguilar et al. (2015), en un estudio sobre apatía, desmotivación, desinterés, desgano y falta de participación en adolescentes mexicanos, reportaron que las actividades escolares eran la primera causa de apatía, desinterés y desgano en hombres y mujeres; y era el principal tipo de actividad en el que preferían no participar. En un estudio sobre deserción en la educación media en Paraguay (Centro de Investigación e Innovación Educativas, 2011), el desinterés (11,54\%) fue, según los docentes, la tercera causa de deserción. 
El análisis de Navarro (2001) sobre marginación escolar también reportó un factor motivacional ("porque la persona no quiso o no le gustó estudiar") como la primera causa (37.4\%) por la cual se realizó el abandono escolar en jóvenes de 15 a 19 años. El estudio de Ruiz-Ramírez, García-Cué y Pérez-Olvera (2014) con estudiantes mexicanos de bachillerato encontró que, después del embarazo y el rendimiento académico, la falta de interés por estudiar (22.9\%) era la tercera causa por la cual los estudiantes abandonaban sus estudios. La baja motivación, con un $8,6 \%$, figuró como el sexto aspecto que manifestaron los estudiantes para justificar su deserción. En el reporte de la situación de la educación media en Colombia, del Ministerio de Educación Nacional (2003), el "no me gusta", como causa de deserción, obtuvo un $30 \%$ de las respuestas de los jóvenes encuestados. Y en otra encuesta realizada por Profamilia en 2000, el "no quise estudiar", con el 20\%, fue la segunda respuesta con mayor porcentaje (Casa Editorial El Tiempo, Corporación Región, Fundación Corona, Fundación Antonio Restrepo Barco, Plan Internacional y UNICEF, 2006).

Porcentajes tan altos (superiores al 20\%) solo se encontraron, además, en el factor académico. Aquí, un 27,2\% de los estudiantes manifestó haberse retirado por no haber querido ser técnico en el programa que se ofrecía en la institución educativa, y un $20,3 \%$ se retiró por que la media técnica no cumplió sus expectativas académicas. Estos porcentajes son consistentes ya que es comprensible que un estudiante se retire de la media técnica si ésta no cumple con sus expectativas académicas porque no es de su interés. Es decir, si el programa que ofrece la institución no es del interés del estudiante, seguramente el programa no cumplirá con sus expectativas. Y, quizás, esta causa explique por qué un $25,7 \%$ de los estudiantes manifestaron haberse retirado de la media técnica por iniciativa propia y sin influencia externa (el mismo razonamiento podría aplicarse para el $17,8 \%$ en el que la media técnica no cumplió sus expectativas laborales).

El aumento de la cobertura de la educación superior en Colombia puede haber generado un cambio en las expectativas académicas de los jóvenes. El mayor número de cupos para acceder a carreras profesionales en las universidades y las instituciones universitarias públicas, las políticas y programas del gobierno nacional sobre el otorgamiento de becas y créditos condonables, y la flexibilidad y disponibilidad en la financiación de los costos de matrícula y manutención (Melo, Ramos y Hernández, 2014) han aumentado las probabilidades de que los jóvenes de estrados socioeconómicos 1 y 2 puedan pensar en la educación superior como una alternativa real de formación profesional y dejen de considerar la educación técnica como la única alternativa. Sin duda, 
la media académica servirá más para su propósito de acceder a la educación superior y las opciones de la media técnica no satisfarán sus expectativas de formación asociadas con un proyecto de vida en el que ser profesional es una alternativa que puede alcanzarse. Según cifras del Sistema de Información de la Educación Para El Trabajo y el Desarrollo Humano (Ministerio de Educación Nacional, 2016), la matrícula, a nivel nacional, descendió de 472.275 estudiantes en 2015 a 391.758 en 2016.

El otro componente del factor académico, en el que los porcentajes de respuesta de los ítems fueron altos (entre el 15\% y el 17\%), fue el relacionado con la exigencia y el rendimiento académico. Un 17,5\% de los estudiantes manifestó que se retiró de la media técnica porque la exigencia era demasiado alta, porque su rendimiento académico general en el bachillerato se afectó negativamente $(17,4 \%)$ o porque tuvo problemas para comprender las competencias o los contenidos en el programa técnico (15\%). La organización de la media técnica tiene dos diferencias substanciales con la media académica. Primero, la intensidad horaria de la media técnica es más alta. Y segundo, la nota aprobatoria en la media técnica es más alta que la nota aprobatoria en la media académica. El desinterés por el programa técnico de la institución y la insatisfacción de las expectativas, sumado a la diferencia en la intensidad horaria y la nota aprobatoria, son elementos que pueden facilitar la comprensión de las razones que tienen algunos estudiantes para abandonar la media técnica y cambiarse a la media académica. La motivación y el interés son aspectos fundamentales del logro académico en la educación básica secundaria (Thornberry, 2003).

En el análisis de los factores asociados con el abandono y la deserción escolar en América Latina, Román (2013) destaca la exigencia académica. Aquí, la exigencia académica habría que entenderla como las diferentes estrategias, métodos y técnicas de evaluación que se derivan de una política evaluativa institucional y que pueden afectar el rendimiento académico (Silvera, 2016). La exigencia académica también hace referencia a todas las actividades contempladas dentro del currículo que están orientadas hacia el cumplimiento de objetivos pedagógicos, el desarrollo de aptitudes y la adquisición de conocimientos. Ya que los objetivos de la media técnica difieren de los de la media académica (el carácter académico tiene como objetivo la adquisición y profundización en conocimientos y habilidades cognitivas relacionadas con la reflexión, el pensamiento crítico, la investigación, la solución de problemas; mientras que el carácter técnico tiene como propósito la preparación para el desempeño laboral en los sectores de la producción y los servicios a través de la formación en la teoría y la práctica desde lo más avanzado de la ciencia y la 
técnica), las actividades contempladas dentro del currículo también difieren, al igual que la exigencia.

Los factores sociales y económicos, como causas del cambio de la media técnica a la media académica, no presentaron porcentajes de consideración. $\mathrm{Si}$ bien es cierto que estos dos factores figuran como algunas de las principales causas de deserción escolar (Ministerio de Educación Nacional, 2011; Landero, 2012), debe recordarse que el presente estudio se concentró en estudiantes que había abandonado la media técnica pero que seguían escolarizados en la media académica. Es decir, que esta población es diferente a la población que deserta y, seguramente, las causas no son las mismas, aunque haya algunas coincidencias. Sí es interesante el 11,2\% que afirmó que se retiró de la media técnica porque salía muy tarde de la institución educativa y esto puede coincidir con el tema de la intensidad horaria que se discutió anteriormente. En la media técnica las jornadas educativas son más extensas. Ninguno de los demás ítems de los factores sociales o económicos superó el 10\% como motivo de abandono de la media técnica.

De manera que los dos factores más relevantes asociados con el cambio de la media técnica a la media académica son el motivacional y el académico, y entre ambos parece haber una lógica que los relaciona. En general, se ha reportado un alto nivel de desinterés y desmotivación hacia las actividades académicas (Aguilar et al., 2015). Si el 29,3\% manifestó no haber recibido información sobre orientación vocacional y el 17,9\% negó haber recibido en noveno grado la información relacionada con el programa de media técnica, no resulta difícil comprender por qué, sumado al desinterés y la desmotivación escolar inherente en algunos estudiantes, se retiran de la media técnica porque ésta no satisface sus expectativas. Es decir, están desinformados y desmotivados (el 25,6\% manifestó no haber ingresado voluntariamente). Y esto explicaría por qué un $25,7 \%$ de los estudiantes informó haberse retirado de la media técnica por iniciativa propia y sin influencia externa. Simplemente dejaron de estudiar un programa que no conocían, que era exigente (académicamente, en recursos económicos y en dedicación de tiempo) y para el cual, posiblemente, no estaban los suficientemente motivados.

Las causas del cambio de la media técnica a la media académica no tendrían por qué coincidir con las causas de la deserción escolar. Si bien es posible que haya algún grado de coincidencia, los dos fenómenos son esencialmente diferentes. Por un lado, el cambio de la media técnica a la media académica es simplemente un cambio de modalidad o de carácter de la educación media, pero no hay abandono del sistema escolar. Lo único que ocurre es que el estudiante, por algunas de las razones que se han presentado y discutido en esta 
investigación, decide no continuar con una modalidad y se cambia a la otra. Por otro lado, la deserción es el retiro del sistema educativo. Por esta razón, probablemente, los factores sociales y económicos estuvieron, prácticamente ausentes (con porcentajes cercanos al 1\%) como posibles causas. Es decir, las razones que motivan a un estudiante a cambiarse de la media técnica a la media académica no son, según los hallazgos del presente estudio, sociales o económicas (con excepción del 11,2\% que afirmó que se retiró de la media técnica porque salía muy tarde de la institución educativa).

\section{Conclusiones}

Luego de analizar factores motivacionales, académicos, sociales y económicos en una muestra de 320 estudiantes de instituciones educativas públicas de la ciudad de Medellín, Colombia, que habían abandonado la media técnica y se habían cambiado a la media académica, la principal conclusión es que es el factor motivacional es el que explica el mayor porcentaje de los casos. De este factor, el no haber recibido orientación vocacional y no haber sido informados adecuadamente sobre los programas técnicos de la institución educativa fueron los principales aspectos asociados con el retiro de la modalidad técnica y el cambio a la modalidad académica. Estos dos aspectos, combinados con el hecho de que un alto porcentaje de estudiantes no querían ser técnicos en el programa que se ofrecía en la institución y la alta exigencia académica, explican por qué un porcentaje considerable se retiró del programa voluntariamente y sin influencia externa. Pero si los estudiantes no recibieron orientación vocacional ni información sobre el programa, ¿por qué se matricularon en esta modalidad? El alto porcentaje de estudiantes que no se matriculó voluntariamente quizás ofrezca una respuesta apropiada.

El aumento de la cobertura de la educación superior en Colombia puede estar generado un cambio en las expectativas académicas de los jóvenes quienes actualmente ven más probabilidades de acceder a la educación superior para obtener un título profesional. En este escenario, es posible que muchos de los jóvenes de estratos socioeconómicos 1, 2 y 3 (el 95\% de la muestra de esta investigación) estén reconsiderando sus opciones formativas y laborales y estén valorando la educación técnica, o la formación para el trabajo, como una alternativa que ya no es necesaria, debido a que es posible acceder a la educación superior y obtener un diploma profesional y, eventualmente, un posgrado. Este "aumento de las expectativas de formación" podría explicar por qué un porcentaje tan alto de los estudiantes se retiró de la media técnica porque ésta no satisfizo sus expectativas. 


\section{Referencias}

Aguilar, Y. P., Valdez, J. L., González, N. I., Rivera, S., Carrasco, C., Gómora, A., Pérez, A. y Vidal, S. (2015). Apatía, desmotivación, desinterés, desgano y falta de participación en adolescentes mexicanos. Enseñanza e Investigación en Psicología, 20(3), 326-336. https://www.redalyc.org/pdf/292/29242800010.pdf

Alcaldía de Medellín. (2014). Análisis de deserción escolar en establecimientos oficiales 2004-2013. Unidad de Análisis Sectorial Subsecretaría de Planeación Secretaría de Educación de Medellín Vicealcaldía de Educación, Cultura, Participación, Recreación y Deporte. Recuperado de: https://www.medellin.gov.co/irj/go/ km/docs/pccdesign/SubportaldelCiudadano_2/PlandeDesarrollo_0_4/Noticias/ Shared\%20Content/Documentos/2014/Boletin0092014DesercionEscolar.pdf

Barrera-Osorio, F., Maldonado, D., Rodríguez, C. (2012). Calidad de la Educación Básica y Media en Colombia: Diagnóstico y Propuestas. Recuperado de: http:// www.calidadeducativasm.com/wp-content/uploads/2015/10/calidad-de-laeducacion-basica-y-media-en-colombia.pdf

Casa Editorial El Tiempo, Corporación Región, Fundación Corona, Fundación Antonio Restrepo Barco, Plan Internacional y UNICEF. (2006). Situación de la educación preescolar, básica, media y superior en Colombia. Bogotá: Gente Nueva Editorial.

Centro de Investigación e Innovación Educativas. (2001). La deserción escolar en Paraguay: características que asume en la educación media. Recuperado de: https:// mec.gov.py/cms_v2/adjuntos/2410

Congreso de Colombia. (19 de julio de 2002). Servicio público de la educación superior en las modalidades de formación técnica profesional y tecnológica. [Ley 749 de 2002]. DO: 44.872. https://www.mineducacion.gov.co/1621/articles-86432_ Archivo_pdf.pdf

Congreso de Colombia. (8 de febrero de 1994). Ley general de educación. [Ley 115 de 1994]. DO: 41.214. https://www.mineducacion.gov.co/1621/articles-85906_ archivo_pdf.pdf

Dimas, P. y Malagón, L. A. (2011). Pertinencia de la educación media técnica en Colombia. Revista Perspectivas Educativas, 4, 51-65. http://revistas.ut.edu.co/ index.php/perspectivasedu/article/download/702/546

Espíndola, E. y León, A. (2002). La deserción escolar en América Latina: un tema prioritario para la agenda regional. Revista Iberoamericana de Educación, 30, 39-62. https://rieoei.org/RIE/article/view/941 
Espínola, V. (2011). Mapa de la Efectividad de la Educación Media en Chile: Factores de gestión asociados a la completación de estudios secundarios. Recuperado de: www. udp.cl/funciones/descargaArchivos.asp?seccion=documentos\&id=131

Ferreyra, H. A., Peretti, G. C., Carandino, E. A., Eberle, M. J., Provinciali, D. M., Rimondino, R. E. y Salgueiro, A. M. (2006) Educación media en Argentina: ¿el problema de los problemas...? Revista Iberoamericana de Educación, 4(39), 1-18. https://rieoei.org/RIE/article/view/2566

García, S., Maldonado, D. y Jaramillo, L. E. (2016). Graduación de la educación media, asistencia e inasistencia a la educación media. Caracterización de la educación media en Colombia. Universidad de los Andes - Escuela de Gobierno Alberto Lleras Camargo. Recuperado de: https://egob.uniandes.edu.co/images/books/DT/DT34.pdf

García, S., Maldonado, D., Acosta, M., Castro, N., Granada, D., Londoño, E., Pérez, J., Rey, C., Rosales, L. y Villalba, H. (2016). Características de la oferta de la educación media en Colombia. Caracterización de la educación media en Colombia. Universidad de los Andes - Escuela de Gobierno Alberto Lleras Camargo. Recuperado de: https://egob.uniandes.edu.co/images/books/DT/DT-33.pdf

Gómez, V. M. (2009). La transición del nivel medio (secundaria superior) al trabajo y la formación postsecundaria en Colombia. Recuperado de: https://www.academia. edu/6121094/_La_transici\%C3\%B3n_del_nivel_medio_secundaria_superior_al_ trabajo_y_la_formaci\%C3\%B3n_postsecundaria_en_Colombia_

Gómez, V. M., Díaz, C. M., Celis, J. E. (2009). El puente está quebrado: aportes a la reconstrucción de la Educación Media en Colombia. Bogotá: Universidad Nacional de Colombia.

Landero, J. F. J. (2012). Deserción en la educación media superior en México. Recuperado de: http://editor.pbsiar.com/upload/PDF/desercion.pdf

Melo, L., Ramos, J., Hernández, P. (2014). La Educación Superior en Colombia: Situación Actual y Análisis de Eficiencia. Borradores de Economía, 808. Recuperado de: http://www.banrep.gov.co/sites/default/files/publicaciones/archivos/ be_808.pdf

Ministerio De Educación Nacional. (16 de diciembre de 2009). Organización, oferta y funcionamiento de la prestación del servicio educativo para el trabajo y el desarrollo humano. [Decreto No. 4904 de 2009]. https://www.mineducacion.gov.co/1621/ articles-216551_archivo_pdf_decreto4904.pdf

Ministerio de Educación Nacional. (2003). Situación de la educación media en Colombia. Recuperado de: http://www.mineducacion.gov.co/1621/articles-85777_archivo_ pdf3.pdf 
Ministerio de Educación Nacional. (2009). Deserción estudiantil en la educación superior colombiana. Metodología de seguimiento, diagnóstico y elementos para su prevención. Recuperado de: http://www.mineducacion.gov.co/sistemasdeinformacion/1735/ articles-254702_libro_desercion.pdf

Ministerio de Educación Nacional. (2011). Encuesta Nacional de Deserción Escolar. Recuperado de: https://www.mineducacion.gov.co/1621/articles-293664_ archivo_pdf_resultados_ETC.pdf

Ministerio de Educación Nacional. (2016a). Sistema De Información Educación Para El Trabajo y Desarrollo Humano. Recuperado de: https://www.mineducacion.gov. co/1759/articles-353023_recurso_7.pdf

Ministerio de Educación Nacional. (2016b). Educación en Colombia. Recuperado de: https://www.mineducacion.gov.co/1759/articles-356787_recurso_1.pdf

Navarro, S. N. (2001). Marginación escolar en los jóvenes, aproximación a las causas de abandono. Revista de información y análisis, 15, 43- 50.

Román, M. (2013). Factores asociados al abandono y la deserción escolar en américa latina: una mirada en conjunto. Revista Iberoamericana sobre Calidad, Eficacia y Cambio en Educación, 11(2), 33-59. https://www.redalyc.org/ pdf/551/55127024002.pdf

Ruiz-Ramírez, R., García-Cué, J. L., Pérez-Olvera, M. A. (2014). Causas y consecuencias de la deserción escolar en el bachillerato: caso Universidad Autónoma de Sinaloa. Ra Ximhai, 10(5), 51-74. https://www.redalyc.org/pdf/461/46132134004.pdf

Sánchez, J. M., Gómez, V. M., Cadavid, G., Urrego, M. I. (2004). Educación media en Colombia: caracterización y propuesta propedéutica para su articulación con la educación superior y el mundo del trabajo. Medellín: Instituto Tecnológico Metropolitano.

Silvera, L. M. (2016). La evaluación y su incidencia en la deserción escolar: ¿Falla de un sistema, de las instituciones educativas, del docente o del estudiante? Revista Educación y Humanismo, 18(31), 313-325. https://revistas.unisimon.edu.co/ index.php/educacion/article/download/2365/2257

Thornberry, G. (2003). Relación entre motivación de logro y rendimiento académico en alumnos de colegios limeños de diferente gestión. Persona, 6, 197-216. https:// dialnet.unirioja.es/descarga/articulo/2879578.pdf

Traversa, F. (2013). Educación, trabajo y nuevas desigualdades. Hacia una economía política del conocimiento para el capitalismo contemporáneo. Nueva Sociedad, 247, 50-69. https://nuso.org/media/articles/downloads/3977_1.pdf 